\title{
On almost complex structures from classical linear connections
}

\begin{abstract}
Let $\mathcal{M} f_{m}$ be the category of $m$-dimensional manifolds and local diffeomorphisms and let $T$ be the tangent functor on $\mathcal{M} f_{m}$. Let $\mathcal{V}$ be the category of real vector spaces and linear maps and let $\mathcal{V}_{m}$ be the category of $m$-dimensional real vector spaces and linear isomorphisms. We characterize all regular covariant functors $F: \mathcal{V}_{m} \rightarrow \mathcal{V}$ admitting $\mathcal{M} f_{m}$-natural operators $\tilde{J}$ transforming classical linear connections $\nabla$ on $m$-dimensional manifolds $M$ into almost complex structures $\tilde{J}(\nabla)$ on $F(T) M=\bigcup_{x \in M} F\left(T_{x} M\right)$.
\end{abstract}

Introduction. All manifolds considered in the paper are assumed to be Hausdorff, finite dimensional, second countable, without boundaries and smooth (of class $C^{\infty}$ ). Maps between manifolds are assumed to be smooth (of class $C^{\infty}$ ).

The category of $m$-dimensional manifolds and local diffeomorphisms is denoted by $\mathcal{M} f_{m}$. The category of vector bundles and vector bundle homomorphisms is denoted by $\mathcal{V B}$. The category of $m$-dimensional real vector spaces and linear isomorphisms is denoted by $\mathcal{V}_{m}$. The category of finite dimensional real vector spaces and linear maps is denoted by $\mathcal{V}$.

The concepts of natural bundles and natural operators can be found in [3].

Let $F: \mathcal{V}_{m} \rightarrow \mathcal{V}$ be a covariant regular functor. The regularity of $F$ means that $F$ transforms smoothly parametrized families of morphisms into smoothly parametrized ones. Let $T: \mathcal{M} f_{m} \rightarrow \mathcal{V B}$ be the tangent functor

2010 Mathematics Subject Classification. 58A20, 58A32.

Key words and phrases. Classical linear connection, almost complex structure, Weil bundle, natural operator. 
sending any $m$-manifold $M$ into the tangent bundle $T M$ of $M$ and any $\mathcal{M} f_{m}$-map $\varphi: M \rightarrow M_{1}$ into the tangent map $T \varphi: T M \rightarrow T M_{1}$. Applying $F$ to fibers $T_{x} M$ of $T M$, one can define a vector natural bundle $F(T)$ over $m$-manifolds of order 1 by

$$
F(T) M=\bigcup_{x \in M} F\left(T_{x} M\right) \text { and } F(T) \varphi=\bigcup_{x \in M} F\left(T_{x} \varphi\right): F(T) M \rightarrow F(T) M_{1}
$$

for any $m$-manifold $M$ and any $\mathcal{M} f_{m}$-map $\varphi: M \rightarrow M_{1}$ between $m$ manifolds. In particular, if $F$ is the identity functor, then $F(T)=T$. If $F V=\otimes^{r} V \otimes \otimes^{q} V^{*}$ then $F(T) M=\otimes^{r} T M \otimes \otimes^{q} T^{*} M$.

It is well known (see e.g. [1]), that if $\nabla$ is a classical linear connection on a manifold $M$, then the tangent bundle $T M$ of $M$ possesses the (canonical) almost complex structure $\tilde{J}^{\nabla}$ such that $\tilde{J}^{\nabla}\left(X^{H}\right)=X^{V}$ and $\tilde{J}^{\nabla}\left(X^{V}\right)=$ $-X^{H}$ for any vector field $X \in \mathcal{X}(M)$ on $M$, where $X^{H} \in \mathcal{X}(T M)$ is the $\nabla$-horizontal lift of $X$ and $X^{V} \in \mathcal{X}(T M)$ is the vertical lift of $X$ to $T M$.

In the present note we study the following problem.

Problem 1. Characterize all covariant regular functors $F: \mathcal{V}_{m} \rightarrow \mathcal{V}$ admitting $\mathcal{M} f_{m}$-natural operators $\tilde{J}$ transforming classical linear connections $\nabla$ on $m$-manifolds $M$ into almost complex structures $\tilde{J}(\nabla)$ on $F(T) M$.

1. Basic definitions. A classical linear connection on a manifold $M$ is a R-bilinear map $\nabla: \mathcal{X}(M) \times \mathcal{X}(M) \rightarrow \mathcal{X}(M)$ such that $\nabla_{f X} Y=f \nabla_{X} Y$ and $\nabla_{X} f Y=X f Y+f \nabla_{X} Y$ for any smooth map $f: M \rightarrow \mathbf{R}$ and any vector fields $X, Y \in \mathcal{X}(M)$ on $M$. Equivalently, a classical linear connection on $M$ is a right invariant decomposition $T L M=H^{\nabla} \oplus V L M$ of the tangent bundle $T L M$ of $L M$, where $L M$ is the principal bundle (with the structural group $G L(m)$ ) of linear frames of $M$ and $V L M$ is the vertical bundle of $L M$, see $[2]$.

A complex structure on a real vector space $W$ is a linear endomorphism $J: W \rightarrow W$ such that $J^{2}=-i d_{W}$.

An almost complex structure on a manifold $N$ is a tensor field $\tilde{J}: T N \rightarrow$ $T N$ on $N$ of type $(1,1)$ (affinor) such that $\tilde{J} \circ \tilde{J}=-i d_{T N}$.

The general concept of natural operators can be found in the fundamental monograph [3]. We need the following particular case of the one, only.

Let $F: \mathcal{V}_{m} \rightarrow \mathcal{V}$ be a covariant regular functor. A $\mathcal{M} f_{m}$-natural operator transforming classical linear connections $\nabla$ on $m$-manifolds $M$ into almost complex structures $\tilde{J}(\nabla): T F(T) M \rightarrow T F(T) M$ on $F(T) M$ is a $\mathcal{M} f_{m^{-}}$ invariant family $\tilde{J}: Q \rightsquigarrow(A C S) F(T)$ of operators

$$
\tilde{J}: Q(M) \rightarrow(A C S)(F(T) M)
$$

for $m$-manifolds $M$, where $Q(M)$ is the set of classical linear connections on $M$ and $(A C S)(N)$ is the set of almost complex structures on $N$. The invariance of $\tilde{J}$ means that if $\nabla \in Q(M)$ and $\nabla_{1} \in Q\left(M_{1}\right)$ are $\varphi$-related 
by an embedding $\varphi: M \rightarrow M_{1}$ (i.e. if $\varphi$ is $\left(\nabla, \nabla_{1}\right)$-affine embedding), then $\tilde{J}(\nabla)$ and $\tilde{J}\left(\nabla_{1}\right)$ are $F(T) \varphi$-related (i.e. $\left.T F(T) \varphi \circ \tilde{J}(\nabla)=\tilde{J}\left(\nabla_{1}\right) \circ T F(T) \varphi\right)$.

Let $F$ be as above. A $\mathcal{V}_{m}$-canonical complex structure on $V \oplus F V$ is a $\mathcal{V}_{m}$-invariant system $J$ of complex structures

$$
J: V \oplus F V \rightarrow V \oplus F V
$$

on (vector spaces) $V \oplus F V$ for $m$-dimensional real vectors spaces $V$. The invariance means that $(\varphi \oplus F \varphi) \circ J=J \circ(\varphi \oplus F \varphi)$ for any linear isomorphism $\varphi: V \rightarrow V_{1}$ between $m$-dimensional vector spaces.

2. The main result. The main result of the present note is the following theorem.

Theorem 1. Let $F: \mathcal{V}_{m} \rightarrow \mathcal{V}$ be a covariant regular functor. The following conditions are equivalent:

(i) There exists a $\mathcal{M} f_{m}$-natural operator $\tilde{J}: Q \rightsquigarrow(A C S) F(T)$.

(ii) There exists a $\mathcal{V}_{m}$-canonical complex structure $J$ on $V \oplus F V$.

Proof. (i) $\Rightarrow$ (ii). Let $\tilde{J}: Q \rightsquigarrow(A C S) F(T)$ be a $\mathcal{M} f_{m}$-natural operator in question. Let $V$ be a $\mathcal{V}_{m}$-object and let $\nabla^{V}$ be the $\left(\mathcal{V}_{m}\right.$-canonical $)$ torsion free flat classical linear connection on $V$. Then the almost complex structure $\tilde{J}\left(\nabla^{V}\right): T F(T) V \rightarrow T F(T) V$ on $F(T) V$ restricts to the complex structure

$$
J:=\tilde{J}\left(\nabla^{V}\right)_{0_{0_{V}}}: T_{0_{0_{V}}} F(T) V \rightarrow T_{0_{0_{V}}} F(T) V
$$

on the tangent space $T_{0_{0_{V}}} F(T) V$ of $F(T) V$ at $0_{0_{V}} \in F(T) V$, where $0_{V}$ is the zero in $V$ and $0_{0_{V}}$ is the zero in $F(T)_{0_{V}} V$. We see that $T_{0_{0_{V}}} F(T) V=$ $V \oplus F V$ modulo the usual identifications. (For, $T V=V \oplus V$, then $F(T) V=$ $V \oplus F V$, and then $T_{0_{0_{V}}} F(T) V=V \oplus F V$.) So,

$$
J: V \oplus F V \rightarrow V \oplus F V
$$

is the complex structure on $V \oplus F V$ for any $\mathcal{V}_{m}$-object $V$. Because of the canonical character of the construction of $J, J$ is $\mathcal{V}_{m}$-canonical.

(ii) $\Rightarrow$ (i). Suppose $J: V \oplus F V \rightarrow V \oplus F V$ is a $\mathcal{V}_{m}$-canonical complex structure. Let $\nabla \in Q(M)$ be a classical linear connection on an $m$ manifold $M$. Let $v \in F(T)_{x} M, x \in M$. Since $F(T)$ is of order $1, F(T) M=$ $L M\left[F(T)_{0} \mathbf{R}^{m}\right]$ (the associated space). Then the $\nabla$-decomposition $T L M=$ $H^{\nabla} \oplus V L M$ induces (in obvious way) the $\nabla$-decomposition $T F(T) M=$ $\tilde{H}^{\nabla} \oplus V F(T) M$. Then we have the identification

$$
T_{v} F(T) M=\tilde{H}_{v}^{\nabla} \oplus V_{v} F(T) M \cong T_{x} M \oplus F(T)_{x} M=T_{x} M \oplus F\left(T_{x} M\right)
$$

canonically depending on $\nabla$, where the equality is the connection decomposition, the identification $\tilde{=}$ is the usual one (namely, $\tilde{H}_{v}^{\nabla}=T_{x} M$ modulo the tangent of the projection of $F(T) M$, and $V_{v} F(T) M=T_{v}\left(F(T)_{x} M\right)=$ 
$F(T)_{x} M$ modulo the standard identification) and the second equality is by the definition of $F(T) M$. We define $\tilde{J}(\nabla)_{\mid v}: T_{v} F(T) M \rightarrow T_{v} F(T) M$ by

$$
\tilde{J}(\nabla)_{\mid v}:=J: T_{x} M \oplus F\left(T_{x} M\right) \rightarrow T_{x} M \oplus F\left(T_{x} M\right)
$$

modulo the above identification $T_{v} F(T) M \tilde{=} T_{x} M \oplus F\left(T_{x} M\right)$. Then $\tilde{J}(\nabla)$ : $T F(T) M \rightarrow T F(T) M$ is an almost complex structure on $F(T) M$. By the canonical character of $\tilde{J}(\nabla)$, the (resulting) family $\tilde{J}: Q \rightsquigarrow(A C S) F(T)$ is a $\mathcal{M} f_{m}$-natural operator.

3. Corollaries. We start with the following two lemmas.

Lemma 1. Let $F: \mathcal{V}_{m} \rightarrow \mathcal{V}$ be a regular covariant functor. Suppose that there is no non-zero $\mathcal{V}_{m}$-canonical linear map $V \rightarrow F V$. Then there is no $\mathcal{V}_{m}$-canonical complex structure on $V \oplus F V$.

Proof. Suppose $J: V \oplus F V \rightarrow V \oplus F V$ is a $\mathcal{V}_{m}$-canonical complex structure. Since there is no non-zero $\mathcal{V}_{m}$-canonical linear map $V \rightarrow F V$, then $J_{\mid V \times\{0\}}$ : $V \rightarrow V$ (modulo the identification $V=V \times\{0\}$ ) is a canonical complex structure on $V$. On the other hand, any $\mathcal{V}_{m}$-canonical linear map $V \rightarrow V$ is a constant multiple of the identity map $i d_{V}$. It is a contradiction.

Lemma 2. Let $r, q$ be non-negative integers. If $r-q \neq 1$, there is no non-zero $\mathcal{V}_{m}$-canonical linear map $k: V \rightarrow \otimes^{r} V \otimes \otimes^{q} V^{*}$.

Proof. Let $k: V \rightarrow \otimes^{r} V \otimes \otimes^{q} V^{*}$ be a $\mathcal{V}_{m^{-}}$canonical linear map. Let $v \in V$. The invariance of $k$ with respect to homotheties $t i d_{V}$ (for $t>0$ ) gives the homogeneity condition $t k(v)=t^{r-q} k(v)$. Then $k(v)=0$ if $r-q \neq 1$.

Then we have the following corollary of Theorem 1.

Corollary 1. Let $r, q$ be non-negative integers. If $r-q \neq 1$, there is no $\mathcal{M} f_{m}$-natural operator $\tilde{J}: Q \rightsquigarrow(A C S) \otimes^{r} T \otimes^{q} T^{*}$ sending classical linear connections $\nabla$ on m-manifolds $M$ into almost complex structures $\tilde{J}(\nabla)$ on $\otimes^{r} T M \otimes \otimes^{q} T^{*} M$.

Proof. We have $F(T) M=\otimes^{r} T M \otimes \otimes^{q} T^{*} M$ for $F V=\otimes^{r} V \otimes \otimes^{q} V^{*}$. By Lemma 2, there is no $\mathcal{V}_{m}$-canonical non-zero linear map $V \rightarrow F V$ if $r-q \neq 1$. So, by Lemma 1 , there is no $\mathcal{V}_{m}$-canonical complex structure on $V \oplus F V=V \oplus\left(\otimes^{r} V \otimes \otimes^{q} V^{*}\right)$ if $r-q \neq 1$. Then there is no $\mathcal{M} f_{m}$-natural operator $\tilde{J}$ in question if $r-q \neq 1$ because of Theorem 1 .

We have also the following two lemmas.

Lemma 3. Let $p$ be a positive integer. Let $F: \mathcal{V}_{m} \rightarrow \mathcal{V}$ be a covariant regular functor given by $F V=V \times \cdots \times V((p-1)$ times of $V)$ and $F \varphi=$ $\varphi \times \cdots \times \varphi((p-1)$ times of $\varphi)$. There is a $\mathcal{V}_{m}$-canonical complex structure on $V \oplus F V$ if and only if $p$ is even. 
Proof. Any $\mathcal{V}_{m}$-canonical map $J: V \times \cdots \times V(p$ times of $V) \rightarrow V \times \cdots \times V$ ( $p$ times of $V$ ) is of the form $J(w)=C w^{T}, w=\left(w_{1}, \ldots, w_{p}\right)$ for some $p \times p$ matrix $C$ with real coefficients. If $J \circ J=-i d_{V}$, then $C^{2}=-i d$, and then $p$ is even. On the other hand, if $p$ is even we have the $\mathcal{V}_{m}$-canonical almost complex structure on $V \times \cdots \times V$ ( $p$ times of $V$ ). Namely, the $\frac{p}{2}$ copies of the canonical almost complex structure on $V \times V,(v, w) \rightarrow(-w, v)$.

Lemma 4 (Lemma 5.1 in [4]). Let $A$ be a p-dimensional Weil algebra and let $T^{A}$ be the corresponding Weil functor. For any classical linear connection $\nabla$ on an $m$-manifold $M$, we have the base-preserving fibred diffeomorphism $I_{\nabla}^{A}: T^{A} M \rightarrow T M \otimes \mathbf{R}^{p-1}$ canonically depending on $\nabla$.

We see that $T M \otimes \mathbf{R}^{p-1}=T M \times_{M} \cdots \times{ }_{M} T M((p-1)$ times of $T M)=$ $F(T) M$, where $F: \mathcal{V}_{m} \rightarrow \mathcal{V}, F V=V \times \cdots \times V((p-1)$ times of $V)$, $F \varphi=\varphi \times \cdots \times \varphi((p-1)$ times of $\varphi)$. So, we have the following corollary of Theorem 1 (and Lemmas 3 and 4 ).

Corollary 2. Let $A$ be a Weil algebra. There exists a $\mathcal{M} f_{m}$-natural operator $\tilde{J}: Q \rightsquigarrow(A C S) T^{A}$ sending classical linear connections $\nabla$ on $m$-manifolds $M$ into almost complex structures $\tilde{J}(\nabla)$ on $T^{A} M$ if and only if $A$ is even dimensional.

Remark 1. If $m$ is odd and the dimension of $A$ is odd, then $T^{A} M$ is odd dimensional and there is no $\mathcal{M} f_{m}$-natural operator in question because of the clear argument. But if $m$ is even and $A$ is odd dimensional, then $T^{A} M$ is even dimensional and theoretically there may exist $\mathcal{M} f_{m}$-natural operators in question. By Corollary 2, such $\mathcal{M} f_{m}$-operators do not exist. For example, there is no $\mathcal{M} f_{m}$-natural operator $\tilde{J}: Q \rightsquigarrow(A C S) T^{2}$ (even if $m$ is even), where $T^{2} M=J_{0}^{2}(\mathbf{R}, M)$ is the second order tangent bundle.

The bundle $L M$ of linear frames of $M$ is open in $T M \times_{M} \cdots \times_{M} T M(m$ times of $T M)$. By Corollary 2, if $m$ is odd, there is an $\mathcal{M} f_{m}$-natural operator $\tilde{J}$ sending classical linear connections $\nabla$ into almost complex structures $\tilde{J}(\nabla)$ on $T M \times_{M} \cdots \times_{M} T M(m$ times of $T M)$. So, we have the following corollary.

Corollary 3. If $m$ is odd, there is a $\mathcal{M} f_{m}$-natural operator $\tilde{J}_{1}$ sending classical linear connections $\nabla$ on $m$-manifolds $M$ into almost complex structures $\tilde{J}_{1}(\nabla)$ on $L M$ such that $\tilde{J}_{1}(\nabla)$ is the restriction of $\tilde{J}(\nabla)$.

Remark 2. For any positive integer $m$ there are many $\mathcal{M} f_{m}$-natural operators $\tilde{J}_{2}: Q \rightsquigarrow(A C S) L$. However, by Corollary 2 , any such $\tilde{J}_{2}(\nabla)$ cannot be extended canonically to an almost complex structure $\tilde{J}(\nabla)$ on the whole $T M \times_{M} \cdots \times_{M} T M(m$ times of $T M)$ if $m$ is even.

\section{REFERENCES}

[1] Dombrowski, P., On the geometry of the tangent bundles, J. Reine Angew. Math. 210 (1962), 73-88. 
[2] Kobayashi, S., Nomizu, K., Foundations of Differential Geometry. Vol. I, J. WileyInterscience, New York-London, 1963.

[3] Kolář, I., Michor, P. W., Slovák, J., Natural Operations in Differential Geometry, Springer-Verlag, Berlin, 1993.

[4] Kurek, J., Mikulski, W. M., On lifting of connections to Weil bundles, Ann. Polon. Math. 103 (3) (2012), 319-324.

Jan Kurek

Institute of Mathematics

Maria Curie-Skłodowska University

pl. M. Curie-Skłodowskiej 1

Lublin

Poland

e-mail: kurek@hektor.umcs.lublin.pl

Received December 19, 2016
Włodzimierz M. Mikulski

Institute of Mathematics

Jagiellonian University

ul. S. Łojasiewicza 6

Cracow

Poland

e-mail: Wlodzimierz.Mikulski@im.uj.edu.pl 